\title{
Structure and Gene Silencing Activities of Monovalent and Pentavalent Cationic Lipid Vectors Complexed with siRNA ${ }^{\dagger}$
}

\author{
Nathan F. Bouxsein, ${ }^{\ddagger}$ Christopher S. McAllister, ${ }^{\S, \|}$ Kai K. Ewert, ${ }^{\ddagger}$ Charles E. Samuel,*,ll and Cyrus R. Safinya*,*,\|l \\ Materials Department and Department of Molecular, Cellular and Developmental Biology, University of California, \\ Santa Barbara, California 93106
}

Received October 13, 2006; Revised Manuscript Received February 12, 2007

\begin{abstract}
Small interfering RNAs (siRNAs) of 19-25 bp mediate the cleavage of complementary mRNA, leading to post-transcriptional gene silencing. We examined cationic lipid (CL)-mediated delivery of siRNA into mammalian cells and made comparisons to CL-based DNA delivery. The effect of lipid composition and headgroup charge on the biophysical and biological properties of CL-siRNA vectors was determined. $\mathrm{X}$-ray diffraction revealed that CL-siRNA complexes exhibited lamellar and inverted hexagonal phases, qualitatively similar to CL-DNA complexes, but also formed other nonlamellar structures. Surprisingly, optimally formulated inverted hexagonal 1,2-dioleoyl-3-trimethylammonium-propane (DOTAP)/1,2dioleoyl-sn-glycero-3-phosphatidylethanolamine (DOPE) CL-siRNA complexes exhibited high toxicity and much lower target-specific gene silencing than lamellar CL-siRNA complexes even though optimally formulated, inverted hexagonal CL-DNA complexes show high transfection efficiency in cell culture. We further found that efficient silencing required cationic lipid/nucleic acid molar charge ratios $\left(\rho_{\text {chg }}\right)$ nearly an order of magnitude larger than those yielding efficiently transfecting CL-DNA complexes. This second unexpected finding has implications for cell toxicity. Multivalent lipids (MVLs) require a smaller number of cationic lipids at a given $\rho_{\text {chg }}$ of the complex. Consistent with this observation, the pentavalent lipid MVL5 exhibited lower toxicity and superior silencing efficiency over a large range in both the lipid composition and $\rho_{\text {chg }}$ when compared to monovalent DOTAP. Most importantly, MVL5 achieved much higher total knockdown of the target gene in CL-siRNA complex regimes where toxicity was low. This property of CL-siRNA complexes contrasts to CL-DNA complexes, where the optimized transfection efficiencies of multivalent and monovalent lipids are comparable.
\end{abstract}

RNA interference (RNAi) ${ }^{1}$ is an evolutionarily conserved post-transcriptional gene-silencing pathway, initially elucidated in Caenorhabditis elegans (1) and in plants $(2,3)$ where long double-stranded RNAs (dsRNAs) mediate sequence specific silencing of gene expression. In mammalian cells, it was discovered that short dsRNAs (19-25 bp, with two 3 '-nucleotide overhangs) $(4-8)$ termed small interfering RNAs (siRNAs), mediate silencing in a sequence-dependent

$\doteqdot$ This research was supported by National Institutes of Health Grants GM-59288, AI-12520, and AI-20611.

* Towhom correspondence shouldbe addressed.C.E.S.: samuel@lifesci. ucsb.edu, (805) 893-3097, fax (805) 893-4724, Molecular, Cellular and Developmental Biology Department, Life Sciences Building 2119. C.R.S.: safinya@mrl. ucsb.edu, (805) 893-8635, fax (805) 893-8797, Materials Research Laboratory, Building 615, Room 2046.

$\doteqdot$ Materials Department.

$\S$ These authors contributed equally to the work presented here

"Department of Molecular, Cellular and Developmental Biology.

${ }^{1}$ Abbreviations: CL, cationic lipid; $\rho_{\text {chg }}$, cationic lipid:nucleic acid molar charge ratio; DOTAP, 1,2-dioleoyl-3-trimethylammoniumpropane; DOPE, 1,2-dioleoyl-sn-glycero-3-phosphatidylethanolamine; DOPC, 1,2-dioleoyl-sn-glycero-3-phosphatidylcholine; $\mathrm{H}_{\mathrm{II}}{ }^{\text {siRNA }}$, inverted hexagonal siRNA phase; $\mathrm{L}_{\alpha}{ }^{\text {siRNA }}$, lamellar siRNA phase; $\sigma_{\mathrm{M}}$, membrane charge density; $\Phi$, mole fraction lipid; MVL, multivalent lipid; NL, neutral lipid; $K_{\mathrm{NS}}$, nonspecific gene silencing; nt, nucleotide; RNAi, RNA interference; siRNA, small interfering RNA; $K_{\mathrm{T}}$, total gene silencing; TE, transfection efficiency; $\delta$, membrane thickness; $\delta_{\text {lipid }}$, thickness of the lipid layer in the membrane; $\delta_{\text {water, }}$ thickness of the water layer between membranes; $\Phi_{\text {lipid }}^{\text {vol }}$, volume fraction of lipid in the membrane. manner. RNAi has led to a surge in research activity aimed at broadly utilizing the technology in functional genomics studies (4) and potentially in therapeutic applications (9).

The specificity of the RNAi machinery has been demonstrated by its ability to discriminate between targets with only one base pair difference (5). Thus, it is conceivable that siRNAs can be designed that selectively knock down the expression of any given gene product when the sequence of the gene is known (10). Such a strategy, for example, provides an alternative to genetic disruptions in model organisms to study loss-of-function phenotypes (10). In addition, therapeutic applications of RNAi are currently being explored in which the targeted RNAs are either endogenous, as in the case of some cancers, or exogenous, such as viral RNAs in the case of infectious diseases like AIDS (9-12).

Applications of RNAi generally require the transfer of either DNA templates that encode short hairpin RNAs (13) or preformed synthetic siRNA duplexes. The utility of RNAi can thus be limited in part by the efficiency and toxicity of the delivery vehicle (10). Mediators of RNAi can be delivered using viral or nonviral methods; furthermore, direct delivery of siRNAs, chemically modified to improve stability and cellular uptake, is possible $(14,15)$. Nonviral delivery most commonly employs cationic lipids or cationic polymers as the vehicle to deliver nucleic acids into the cell $(16-23)$. While these methods generally avoid the immunogenicity 
and integration issues sometimes associated with viral techniques (24), they are not optimized to achieve the delivery efficiency seen with virus-based vectors. Because cationic lipid-based transfection offers the potential to deliver siRNA for both functional genomics and therapeutic purposes (25), it is of utmost importance to gain a better understanding of both the physical characteristics and biological activities of CL-siRNA complexes. Understanding the mechanism of action of these complexes will allow for the optimization of lipid carriers for siRNA molecules, thereby making them a viable alternative to virus-based delivery.

For CL-DNA complexes, which deliver long dsDNA into cells, structures and transfection trends have been elucidated in considerable detail $(18,19)$, demonstrating that both membrane composition and lipid to DNA ratio dramatically affect the complex structure and transfection efficiency (TE; a measure of the expression of an exogenous gene that is transferred) $(18,19,26)$. We now have looked for similar structural and functional characteristics in CL-siRNA complexes with the long-term goal of optimizing silencing efficiency (a measure of post-transcriptional, specific silencing of the gene targeted by the transferred siRNA) of CLsiRNA complexes in mammalian cells. We found that strategies aimed at optimizing silencing efficiency of $\mathrm{CL}-$ siRNA complexes have some similarities to but also significant differences from those aimed at optimizing TE of CL-DNA complexes.

To quantify the gene silencing activity of CL-siRNA complexes, we measured the effect of lipid composition and molar charge ratio ( $\rho_{\text {chg }}$; cationic lipid:nucleic acid) on both the target gene knockdown and nonspecific gene silencing (with the latter correlating to cytotoxicity). We prepared CLsiRNA complexes with monovalent 1,2-dioleoyl-3-trimethylammonium-propane (DOTAP) or pentavalent MVL5 cationic lipids (26) combined with one of two commonly used neutral lipids (NLs): 1,2-dioleoyl-sn-glycero-3-phosphatidylcholine (DOPC) or 1,2-dioleoyl-sn-glycero-3-phosphatidylethanolamine (DOPE). Changing the type of neutral lipid enabled us to elucidate its contribution to structure and silencing efficiency for the $\mathrm{CL}$-siRNA complexes. The delivered siRNA targeted the firefly luciferase mRNA and consisted of a 21 nt long siRNA (Dharmacon, target sequence CUUACGCUGAGUACUUCGA).

Surprisingly, CL-siRNA complexes exhibited structures that were generally similar to CL-DNA complexes, but some important aspects of their transfection properties differed dramatically from their CL-DNA counterparts. We found that efficient delivery of siRNAs into cells in culture required a molar charge ratio $\left(\rho_{\text {chg }}\right)$ nearly an order of magnitude larger than that needed for $\mathrm{CL}-\mathrm{DNA}$ complexes. This larger $\rho_{\text {chg }}$, necessary for efficient $\mathrm{CL}-$ siRNA silencing, resulted in the use of larger amounts of cationic lipid. Consequently, toxicity becomes an important issue to consider in some composition regimes. Our finding also implies that cationic multivalent lipids (MVLs) should be less toxic compared to monovalent cationic lipids, because a smaller number of MVLs are required for a given $\rho_{\text {chg }}$ of the complex. Indeed, as we show, for lamellar complexes, the multivalent MVL5 (5+) was found to exhibit superior silencing efficiency over a wide range of lipid compositions and molar charge ratios compared to monovalent DOTAP. MVL5 was also less toxic than DOTAP.
We observed that the NL component of the complex, DOPE or DOPC, significantly impacted nonspecific gene silencing, toxicity, and the silencing efficiency of CL-siRNA complexes. Previously we have shown (27) that when DOPE is substituted for DOPC in DOTAP/DOPC-DNA complexes, an inverted hexagonal phase is formed, and that the inclusion of DOPE improves the transfection efficiency of these complexes in the low membrane charge density $\left(\sigma_{\mathrm{M}}\right.$; lipid membrane charge per unit area) regime (28). DOTAP/ DOPE-siRNA complexes also formed an inverted hexagonal phase. However, despite the common use of DOPE as a helper lipid for DNA delivery, it was highly toxic to cells when used at concentrations required for efficient siRNA delivery, and thus, the addition of DOPE did not enhance silencing efficiency.

\section{MATERIALS AND METHODS}

Liposome Preparation. DOTAP (molecular weight (MW) $=698.55 \mathrm{~g} / \mathrm{mol})$, DOPC $(\mathrm{MW}=786.15 \mathrm{~g} / \mathrm{mol})$, and DOPE $(\mathrm{MW}=740.01 \mathrm{~g} / \mathrm{mol})$ were purchased from Avanti Polar Lipids (Alabaster, AL). MVL5 (MW = $1552.7 \mathrm{~g} / \mathrm{mol}$ ) was synthesized according to the procedure previously described (29). Liposomes were prepared by volumetrically combining solutions of CLs and NLs in either chloroform (for DOTAP) or chloroform/methanol (v/v, 9:1, for MVL5) solutions. The lipid solutions were dried under a stream of nitrogen and then placed in high vacuum to ensure complete removal of the solvent. The dried lipid mixtures were hydrated at 37 ${ }^{\circ} \mathrm{C}$ for $2 \mathrm{~h}$ in sterile $(18.2 \mathrm{M} \Omega$ ) water to a final concentration of $30 \mathrm{mM}$ for X-ray samples or $1 \mathrm{mM}$ for transfection and cytotoxicity experiments. All aqueous lipid solutions were sonicated to clarity in a bath sonicator at $40{ }^{\circ} \mathrm{C}$ for $4 \mathrm{~h}$ prior to use and stored at $4{ }^{\circ} \mathrm{C}$.

$X$-ray Samples. CL-siRNA complexes were prepared by mixing $50 \mu \mathrm{g}$ of $21 \mathrm{nt}$ long siRNA (Dharmacon, target sequence CUUACGCUGAGUACUUCGA with 3'-nucleotide overhangs, dTdT) at $10 \mathrm{mg} / \mathrm{mL}$ with the desired amount of $30 \mathrm{mM}$ cationic liposomes in a $1.5 \mathrm{~mm}$ quartz capillary (Hilgenberg Glas, Germany). An equal volume of Opti-MEM (Invitrogen, Carlsbad, CA) was then added to the siRNA/ liposome solution. The quartz capillaries were spun at $1000 \mathrm{rpm}$ for $5 \mathrm{~min}$, sealed, and stored at $4{ }^{\circ} \mathrm{C}$. Highresolution X-ray diffraction experiments were conducted at the Stanford Synchrotron Radiation Laboratory beamline 4-2. Complexes were not oriented, producing powder diffraction patterns collected on a MarCCD (Mar USA, Evanston, IL), and the data were radially averaged.

For lipid only controls, lipid powders were weighed out in the appropriate ratios and then mixed with water to form $40 \mathrm{wt} \%$ water solutions. These mixtures where centrifuged at $10000 \mathrm{rpm}$ for $10 \mathrm{~min}$, then mixed with a Teflon spatula. After mixing, the samples were again spun at $10000 \mathrm{rpm}$ for $10 \mathrm{~min}$. The mixtures were then allowed to incubate at room temperature for 5 days. The mixtures were then transferred into quartz capillaries and sealed. High-resolution $\mathrm{X}$-ray diffraction experiments were conducted as stated above.

Cell Culture Experiments. Mouse fibroblast L-cells were cultured in Dulbecco's modified Eagle's medium (DMEM, GIBCO) with 5\% (v/v) fetal bovine serum (GIBCO) and 1\% $(\mathrm{v} / \mathrm{v})$ penicillin and streptomycin $(\mathrm{GIBCO})$ at $37^{\circ} \mathrm{C}$ in a 
humidified atmosphere with $5 \% \mathrm{CO}_{2}$. Cells were seeded into 24-well plates at 85000 cells per well. Luciferase DNA plasmids [pGL3 firefly (Promega, Madison WI) and phRL Renilla (Promega)] were mixed with Lipofectamine 2000 (L2000, Invitrogen) in Opti-MEM for $30 \mathrm{~min}$ to form complexes. A ratio of $380 \mathrm{ng}$ of pGL3 to $20 \mathrm{ng}$ of phRL to $1 \mu \mathrm{L}$ of L2000 per $200 \mu \mathrm{L}$ of Opti-MEM was used for the complexes. $200 \mu \mathrm{L}$ of the complex solution was then added to each well $18 \mathrm{~h}$ after seeding. After a $3 \mathrm{~h}$ incubation, wells were washed once with Opti-MEM and DMEM maintenance media was then added to the wells. Three hours later, the cells were again washed with Opti-MEM and CL-siRNA complexes were added to the cells.

All CL-siRNA complexes contained $100 \mathrm{nM}(0.266 \mu \mathrm{g})$ siRNA targeting the firefly luciferase (sequence identical to that used for X-ray experiments). The amount of cationic lipid was determined by $\rho_{\text {chg }}$, and lipid composition was determined by the mole fraction $\mathrm{NL}\left(\Phi_{\mathrm{NL}}\right)$ present in the liposomes. CL-siRNA complexes were diluted in OptiMEM to a final volume of $0.25 \mathrm{~mL}$ and incubated for $30 \mathrm{~min}$ prior to their addition to the cells. After incubation for $3 \mathrm{~h}$, the cells were again washed with Opti-MEM and maintenance medium was added. Cells were harvested $20 \mathrm{~h}$ later in $150 \mu \mathrm{L}$ of Passive Lysis Buffer (Promega). $20 \mu \mathrm{L}$ of lysate extract was used for the Dual Luciferase Assay as described by Promega. An Autoluminat LB953 luminometer (EG\&G Berthold) was used to measure the relative light units (RLU) from the luminescence assay. All siRNA silencing values represent the average of two to four independent experiments, each performed in duplicate. Luciferase expression for each well was normalized to control wells, which differ from experimental wells only in that they are treated with Opti-MEM instead of CL-siRNA complexes during the third $3 \mathrm{~h}$ incubation step.

Cytotoxicity. Mouse fibroblast L-cells were seeded in 96well plates at 15000 cells per well $18 \mathrm{~h}$ prior to treatment. $\mathrm{CL}$-siRNA complexes were prepared as above, but scaled to $0.0532 \mu \mathrm{g}$ of siRNA. The complexes or corresponding liposomes were added to the wells and incubated for $3 \mathrm{~h}$. After incubation, $75 \mu \mathrm{L}$ of CytoTox-ONE Homogeneous Membrane Integrity Assay (Promega) and $35 \mu \mathrm{L}$ of OptiMEM were added to each well. Cytotoxicity was measured through the quantification of released lactate dehydrogenase (LDH) from cells with damaged membranes. LDH released into the culture medium was measured with a 10 min coupled enzymatic assay using CytoTox-One, which results in the conversion of resazurin (the substrate supplied by CytoToxOne) into resorufin. Resorufin excites at $560 \mathrm{~nm}$ and emits at $590 \mathrm{~nm}$. Fluorescence was quantified on a Bio-Rad PharosFX Molecular Imager System. As controls, some wells were left untreated (no complexes or liposomes) to measure background (minimum) fluorescence, while some wells were left untreated, but lysed after the $3 \mathrm{~h}$ incubation to measure the maximum amount of LDH, i.e., the maximum fluorescence. The values for the minimum, maximum, and sample fluorescence were used to determine the percent toxicity.

\section{RESULTS AND DISCUSSION}

$X$-ray Structure Studies. X-ray diffraction (XRD) scans of $\mathrm{CL}-$ siRNA complexes are shown in Figure $1 \mathrm{~A}-\mathrm{C}$. Figure 1A shows XRD scans of DOTAP/DOPE-siRNA
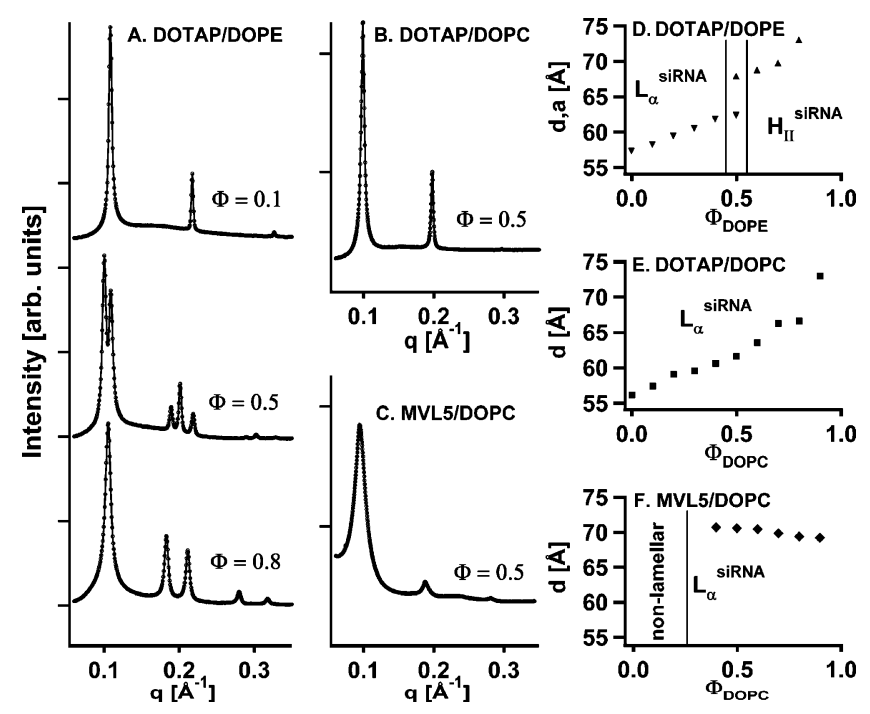

FIGURE 1: $(\mathrm{A}-\mathrm{C})$ Synchrotron $\mathrm{X}$-ray diffraction data of $\mathrm{CL}-$ siRNA complexes showing lamellar $\left(\mathrm{L}_{\alpha}{ }^{\text {siRNA }}\right)$ and inverted hexagonal $\left(\mathrm{H}_{\mathrm{II}}{ }_{\mathrm{SiRNA}}\right.$ ) phases. (A) X-ray diffraction data for DOTAP/ DOPE-siRNA complexes at $\rho_{\text {chg }}=10$. (Top) $\Phi_{\text {DOPE }}=0.1$ in $\mathrm{L}_{\alpha}$ siRNA phase, (middle) $\Phi_{\mathrm{DOPE}}=0.5$ in coexisting $\mathrm{L}_{\alpha}$ siRNA and $\mathrm{H}_{\text {II }}^{\text {siRNA }}$ phases, (bottom) $\Phi_{\text {DOPE }}=0.8$ in single phase $\mathrm{H}_{\mathrm{II}}{ }^{\text {siRNA }}$ phase. (B) X-ray diffraction data for DOTAP/DOPC-siRNA complexes at $\rho_{\text {chg }}=10$ and $\Phi_{\mathrm{DOPC}}=0.5$ in the $\mathrm{L}_{\alpha}{ }^{\text {siRNA }}$ phase. (C) X-ray diffraction data for MVL5/DOPC complexes at $\rho_{\text {chg }}=10$ and $\Phi_{\text {DOPC }}$ $=0.5$ in the $\mathrm{L}_{\alpha}$ siRNA phase. (D) Measured layer spacings $d$ (inverted triangles $\left.=\mathrm{L}_{\alpha}{ }^{\text {siRNA }}\right), a$ (triangles $=\mathrm{H}_{\mathrm{II}}{ }^{\text {siRNA }}$ ) for DOTAP/DOPE complexes at $\rho_{\text {chg }}=10$. (E) Measured layer spacings $d$ (squares $=$ $\mathrm{L}_{\alpha}{ }^{\text {siRNA }}$ ) for DOTAP/DOPC complexes at $\rho_{\text {chg }}=10$. (F) Measured layer spacings $d$ (diamonds $=\mathrm{L}_{\alpha}{ }^{\text {siRNA }}$ ) for MVL5/DOPC complexes at $\rho_{\text {chg }}=10$.

complexes at $\rho_{\text {chg }}=10$ and mole fraction DOPE $\left(\Phi_{\mathrm{DOPE}}\right)=$ $0.1,0.5$, and 0.8 , where a structural transition occurs from a lamellar $\left(\mathrm{L}_{\alpha}{ }^{\text {siRNA }}\right)$ phase to an inverted hexagonal $\left(\mathrm{H}_{\mathrm{II}}{ }^{\text {siRNA }}\right)$ phase with a region of coexistence in between. This trend is similar to that observed for complexes formed with DNA (27), differing only in the location of the compositional phase boundaries. The sharp [00L] peaks, visible in Figure 1A at $\Phi_{\text {DOPE }}=0.1$, result from the periodically spaced lamellar structure composed of siRNA layers sandwiched between cationic lipid bilayers with the interlayer spacing $d=\delta_{\text {lipid }}$ $+\delta_{\text {siRNA }}$ (thickness of the membrane plus the monolayer of siRNA and water) $=2 \pi / q_{001}=57 \AA$ (30) (Figure 2, top, labeled $\mathrm{L}_{\alpha}{ }^{\text {siRNA }}$ ). The sharp peaks visible in Figure 1A at $\Phi_{\text {DOPE }}=0.8$ index to a two-dimensional inverted hexagonal lattice with a unit cell spacing $a=4 \pi / \sqrt{ } 3 q_{10}=70 \AA$. This structure is composed of siRNA molecules inserted within cylindrical lipid nanotubules with the cylinders arranged on a hexagonal lattice (27) (Figure 2, bottom, labeled $\mathrm{H}_{\mathrm{II}}{ }^{\text {siRNA }}$ ). Figure 1D shows the interlayer spacings and unit cell spacings ( $d$ and $a$, respectively) and the phase transition boundaries as a function of $\Phi_{\text {DOPE }}$ for DOTAP/DOPEsiRNA complexes at $\rho_{\text {chg }}=10$. The $\mathrm{L}_{\alpha}{ }^{\text {siRNA }}$ to $\mathrm{H}_{\text {II }}{ }^{\text {siRNA }}$ transition for siRNA complexes occurred over a narrow range of compositions (Figure 1D), in contrast to similar DNA complexes (27).

DOTAP/DOPC-siRNA complexes form the lamellar structure at all $\Phi_{\text {DOPC }}$ between 0 and 0.9 at $\rho_{\text {chg }}=10$. A representative XRD scan for $\Phi_{\mathrm{DOPC}}=0.5$ is shown in Figure 1B. This behavior is similar to that of DOTAP/ DOPC-DNA complexes (30). The interlayer spacing, $d$, is plotted as a function of $\Phi_{\text {DOPC }}$ in Figure 1E. The dramatic 


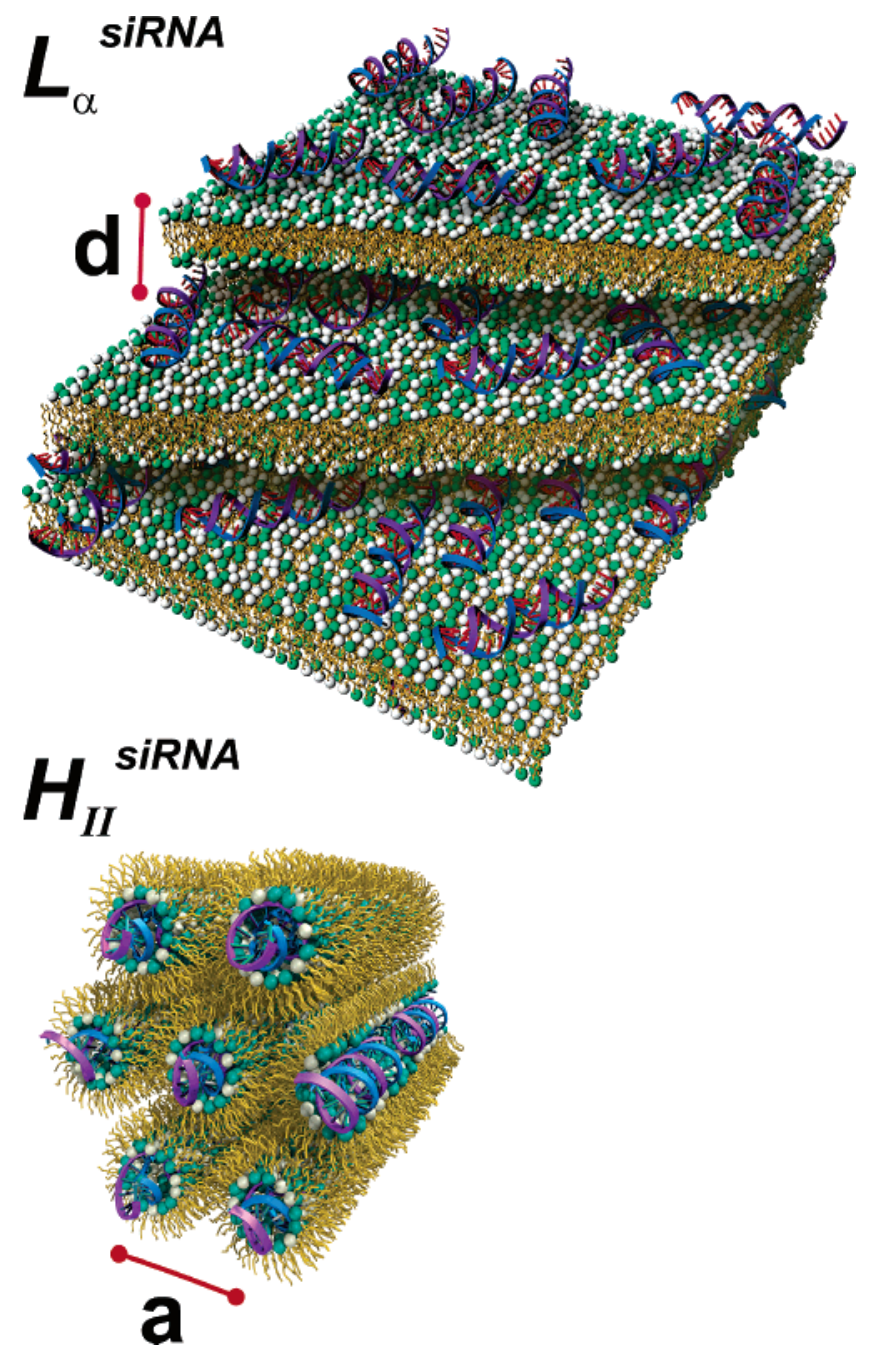

FIGURE 2: Schematic sketch of the lamellar $\mathrm{L}_{\alpha}{ }^{\text {siRNA }}$ (top) and $\mathrm{H}_{\text {II }}$ siRNA (bottom) complexes as described in the text. In the $\mathrm{L}_{\alpha}$ siRNA phase, a partial bilayer has been removed, exposing a monolayer of the electrostatically adhered short siRNA nucleic acids ( $d$ and $a$ as in Figure 1).

increase in the interlayer separation at high amounts of DOPC is consistent with a "pinched lamellar" phase (31), where the small siRNA molecules associate with regions of locally enriched DOTAP to form anchor points along the bilayer. Large pockets of water, stabilized by hydration repulsion in regions of the membrane rich in DOPC, are contained between regions of the membrane rich in DOTAP "pinched" electrostatically by the siRNA.

Figure 1C shows an XRD pattern of a MVL5/DOPCsiRNA complex that exhibited the lamellar structure for $\Phi_{\text {DOPC }}=0.5$ and $\rho_{\text {chg }}=10$. The lamellar structure was observed for all $\Phi_{\mathrm{DOPC}}>0.3$, similar to previous findings $(19,26)$ for MVL5/DOPC-DNA complexes. For $\Phi_{\text {DOPC }} \leq$ 0.3 , a nonlamellar phase was present, the structure of which remains to be determined. Figure $1 \mathrm{~F}$ shows the interlayer spacing, $d$, of MVL5/DOPC-siRNA complexes as a function of $\Phi_{\mathrm{DOPC}}$. In contrast to DOTAP/DOPC - siRNA complexes, $d$ remained locked at a single value for MVL5 complexes, independent of $\Phi_{\text {DOPC }}$. A likely explanation is that MVL5 and DOPC have headgroup hydration layers of comparable size, allowing for the interlayer spacing to appear unchanged with the addition of DOPC.
The peak widths observed for $\mathrm{CL}-$ siRNA complexes were typically narrower than those of CL-DNA complexes, implying a higher degree of local ordering. Time-resolved XRD studies revealed that CL-siRNA complexes achieve their equilibrium structure in a matter of hours, while CLDNA complexes require days $(27,30)$. These observations may be explained by the larger diffusion constant of the short siRNA molecules. The observation of a very weak, broad peak at $q=0.23 \AA$ in Figure $1 \mathrm{C}$ is consistent with our expectation of isotropic liquid like correlation behavior of the siRNA rods (length $\sim 50 \AA$ and diameter $\sim 26 \AA$ for A-form dsRNA) between bilayers and contrasts to the strong correlation peaks seen with CL-DNA complexes (30).

Lipid mixture controls of DOTAP/DOPE, DOTAP/DOPC, and MVL5/DOPC in $40 \mathrm{wt} \%$ water were prepared in the absence of siRNA. SAXS diffractions of these highly concentrated samples are shown in Figure 3. This weight fraction was chosen because lipids are normally in their one phase region at low water contents, which enabled the measurement of the membrane thickness for different neutral lipid volume fractions.

The DOTAP/DOPE mixtures (Figure 3A) showed a transition from the purely lamellar $\mathrm{L}_{\alpha}$ phase to the inverted hexagonal $\mathrm{H}_{\mathrm{II}}$ phase as a function of increasing DOPE concentration. This trend is seen in Figure 3A top, middle, and bottom. At $\Phi_{\mathrm{DOPE}}=0.5$ (top), the three peaks index to the lamellar [00L] structure with $d=2 \pi / q_{001}=53.1 \AA=$ the membrane thickness plus the thickness of the water layer $\left(d=\delta_{\text {lipid }}+\delta_{\text {water }}\right)$. The membrane thickness (including the lipid headgroup) $\delta_{\text {lipid }}=\delta_{\text {lipid tails }}+\delta_{\text {lipid heads }}$ can be calculated from the volume fraction of lipid: $\delta_{\text {lipid }}=\Phi_{\text {lipid }}^{\text {vol }} d$ and for $\Phi_{\text {DOPE }}=0.5, \delta_{\text {lipid }}=33.1 \AA$. Figure 1A (middle) shows that siRNA complexes at $\Phi_{\mathrm{DOPE}}=0.5$ were phase coexistent indicating that the inclusion of nucleic acid shifts the phase boundaries. The diffraction peaks in Figure 3A (middle and bottom) index to the inverse hexagonal $\mathrm{H}_{\mathrm{II}}$ phase with the unit cell spacing, $a=65.7 \AA$ for $\Phi_{\mathrm{DOPE}}=0.85$ and $50.1 \AA$ for $\Phi_{\mathrm{DOPE}}=1$.

For 40 wt \% water mixtures, both DOTAP and DOPC lipids exhibited the lamellar phase, indicated by the diffraction scans shown in Figure $3 \mathrm{~B}$ (DOTAP, $\Phi_{\mathrm{DOPC}}=0$, top, and DOPC, $\Phi_{\mathrm{DOPC}}=1$, bottom) with the expected [00L] peaks. For DOTAP, $d=54.4 \AA$ and $\delta_{\text {lipid }}=34.1 \AA$, while for DOPC, $d=56.9 \AA$ and $\delta_{\text {lipid }}=33.8 \AA$.

MVL5/DOPC mixtures at $40 \mathrm{wt} \%$ water, shown in Figure $3 \mathrm{C}$, also exhibited lamellar $\mathrm{L}_{\alpha}$ phase behavior indicated by the expected [00L] peaks (at lower lipid concentrations, $>90$ wt $\%$ water, the lipids form micelles). MVL5 mixtures (Figure $3 \mathrm{C}$, top) with $\Phi_{\mathrm{DOPC}}=0$ and $\Phi_{\text {lipid }}^{\mathrm{vol}}=0.60 \mathrm{had}$ measured $d=54 \AA$ and $\delta_{\text {lipid }}=\delta_{\text {lipid tails }}+\delta_{\text {lipid heads }}=$ $32.5 \AA$. MVL5 mixtures (Figure 3C, middle) with $\Phi_{\text {DOPC }}=0.25$ and $\Phi_{\text {lipid }}^{\mathrm{vol}}=0.597$ had calculated $d=58.3 \AA$ and $\delta_{\text {lipid }}=35 \AA$. MVL5 mixtures (Figure 3C, bottom) with $\Phi_{\text {DOPC }}=0.75$ and $\Phi_{\text {lipid }}^{\text {vol }}=0.595$ had calculated $d=86.5 \AA$ and $\delta_{\text {lipid }}=51.5 \AA$ (including the headgroup). Assuming minimal change in the length of the headgroup, it is reasonable to conclude that the decrease in the $d$ spacing seen with increasing MVL5 arises from compression of the lipid tails in order to accommodate the lamellar structure.

Cell Culture Transfection Studies. To carry out the gene silencing efficiency experiments, mouse L-cells were first 

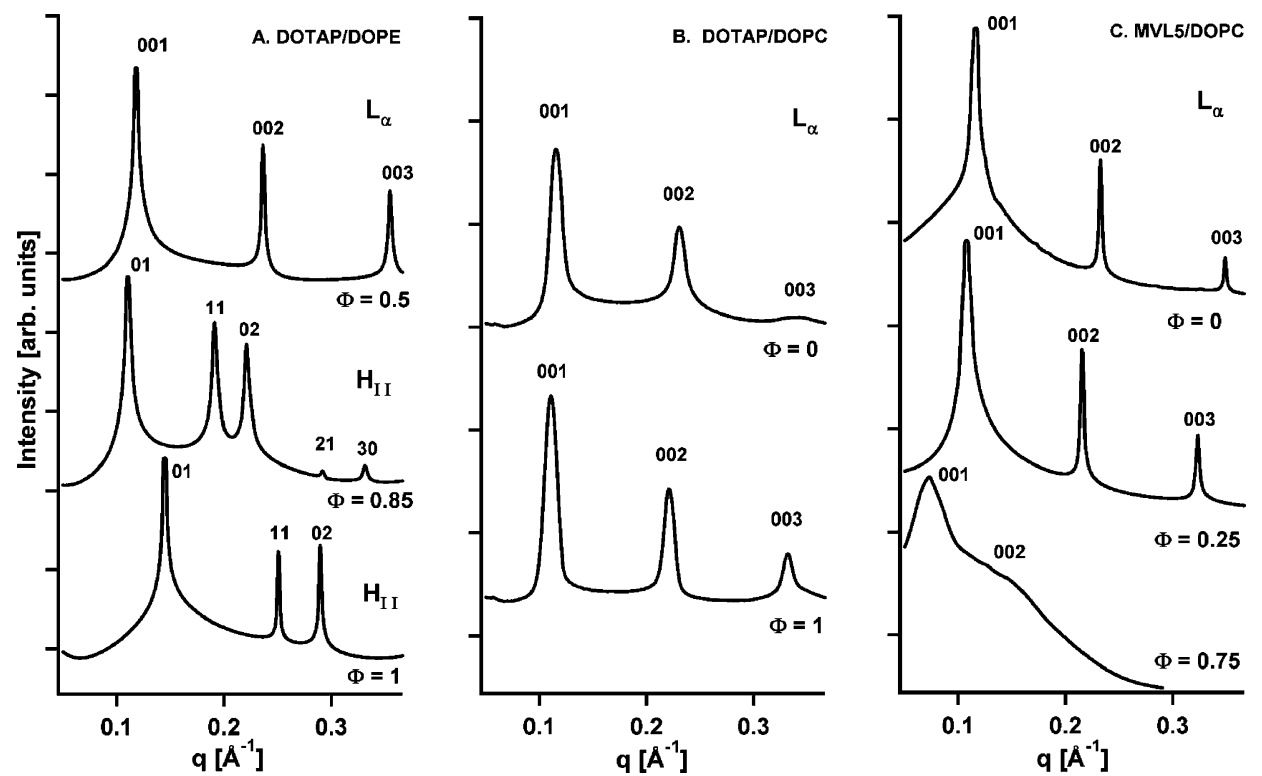

FIGURE 3: Synchrotron X-ray diffraction data of lipid mixtures in $40 \mathrm{wt} \%$ water and no added siRNA. (A) DOTAP/DOPE mixtures. At $\Phi_{\mathrm{DOPE}}=0.5$ (A, top) the lipid mixture indexes to a perfect lamellar, $\mathrm{L}_{\alpha}$ structure. At $\Phi_{\mathrm{DOPE}}=0.85\left(\mathrm{~A}\right.$, middle) and $\Phi_{\mathrm{DOPE}}=1(\mathrm{~A}$, bottom, pure DOPE) the mixtures index to an inverted hexagonal, $\mathrm{H}_{\mathrm{II}}$ structure. (B) $40 \mathrm{wt} \%$ water DOTAP mixture (B, top) and $40 \mathrm{wt} \%$ water DOPC mixture $\left(B\right.$, bottom) both exhibit the $\mathrm{L}_{\alpha}$ phase. $(\mathrm{C}) 40 \mathrm{wt} \%$ water MVL5/DOPC mixtures at $\Phi_{\mathrm{DOPC}}=0,0.25$, and $0.75(\mathrm{C}$, top, middle, and bottom, respectively) indexed to the $\mathrm{L}_{\alpha}$ phase.

cotransfected with plasmids encoding the firefly (FF) and Renilla (RL) luciferases. The cells were then either transfected with CL-siRNA complexes (as a function of $\Phi_{\mathrm{NL}}$ and $\rho_{\text {chg }}$ ) with siRNA targeting the mRNA for FF luciferase or used as controls. A dual luciferase assay was used to measure the expression of $\mathrm{FF}$ (denoted $\mathrm{FF}\left(\Phi_{\mathrm{NL}}, \rho_{\mathrm{chg}}\right)$ ) and RL (denoted $\operatorname{RL}\left(\Phi_{\mathrm{NL}}, \rho_{\text {chg }}\right)$ ) luciferase activities. FF and RL expression levels were also measured for a similar number of cells that were not transfected with the $\mathrm{CL}-$ siRNA complexes, yielding the controls $\mathrm{FF}_{\text {cont }}$ and $\mathrm{RL}_{\text {cont }}$. Thus, by measuring $\operatorname{FF}\left(\Phi_{\mathrm{NL}}, \rho_{\text {chg }}\right), \mathrm{FF}_{\text {cont }}, \operatorname{RL}\left(\Phi_{\mathrm{NL}}, \rho_{\text {chg }}\right)$, and $\mathrm{RL}_{\text {cont }}$, one readily obtains the total normalized target gene knockdown $K_{\mathrm{T}}=1-\mathrm{FF}\left(\Phi_{\mathrm{NL}}, \rho_{\text {chg }}\right) / \mathrm{FF}_{\text {cont }}$, and the normalized nonspecific gene knockdown $K_{\mathrm{NS}}=1-\operatorname{RL}\left(\Phi_{\mathrm{NL}}, \rho_{\text {chg }}\right) / \mathrm{RL}_{\text {cont }}$.

The total knockdown $\left(K_{\mathrm{T}}\right)$ includes the sequence-specific silencing of the target FF luciferase by the siRNA together with any nonspecific inhibitory effects contributing to suppression of protein synthesis. The nonspecific knockdown $\left(K_{\mathrm{NS}}\right)$ measures the sequence-independent protein reduction mediated by $\mathrm{CL}-$ siRNA complexes due to cytotoxicity. $K_{\mathrm{NS}}$ is determined using the expression of the nontargeted $\mathrm{RL}$ luciferase in cells transfected with CL-siRNA complexes containing siRNA that targets the distinctly different FF luciferase mRNA. As we show below, $K_{\mathrm{NS}}$ is directly related to cytotoxicity and appears to be dominated by the cationic liposome component. Optimal gene silencing would correspond to $K_{\mathrm{T}}$ approaching 1 and $K_{\mathrm{NS}}$ approaching zero, where the sequence-specific gene silencing contribution to $K_{\mathrm{T}}$ dominates.

The total knockdown, $K_{\mathrm{T}}$, and nonspecific knockdown, $K_{\mathrm{NS}}$, as a function of $\rho_{\mathrm{chg}}$ were measured at $\Phi_{\mathrm{NL}}=0,0.1$, $0.2,0.3,0.4,0.5,0.6,0.7,0.8$, and 0.9 . Figure 4 shows plots of $K_{\mathrm{T}}$ and $K_{\mathrm{NS}}$ for MVL5/DOPC-siRNA (left), DOTAP/ DOPC - siRNA (middle), and DOTAP/DOPE-siRNA (right) complexes, at $\Phi_{\mathrm{NL}}=0.1$ (bottom) and 0.4 (top). Similar behavior was observed at $\Phi_{\mathrm{NL}}=0,0.2,0.3,0.5,0.6$, and 0.7 (data not shown). For $\Phi_{\mathrm{NL}} \geq 0.8$, the behavior was dominated by large $K_{\mathrm{NS}}$ due to a combination of cytotoxicity and low lipid membrane charge density $\left(\sigma_{\mathrm{M}}\right)$ resulting in a weak electrostatic attraction between the complex and the cell (data not shown). The data show that, for the lamellar siRNA complexes of pentavalent MVL5/DOPC, the nonspecific knockdown remains nearly constant and low with $K_{\mathrm{NS}}<0.15$ for $2.8 \leq \rho_{\text {chg }} \leq 20$, while $K_{\mathrm{T}}$ exhibits a rapid nonlinear growth to approximately 0.9 , indicative of significant sequence-specific gene silencing (Figure 4, left). In contrast, such a region of relatively high $K_{\mathrm{T}}$ and low $K_{\mathrm{NS}}$ was not observed for the lamellar phases of monovalent DOTAP/DOPC - siRNA complexes at $\Phi_{\mathrm{NL}}=0.4$ (or $\Phi_{\mathrm{NL}}=$ 0.1 ), where $K_{\mathrm{T}}$ varied slowly between 0.4 and 0.55 for $5 \leq$ $\rho_{\text {chg }} \leq 15$ with $K_{\mathrm{NS}}$ approximately $=0.2$ (Figure 4, middle). Furthermore, for DOTAP/DOPE-siRNA complexes, substantial nonspecific knockdown $K_{\mathrm{NS}}$ (related to cell toxicity) is observed even at a low $\rho_{\text {chg }}$ of approximately 5 (Figure 4, right). These data are in striking contrast to that of similar, optimally formulated DNA complexes containing DOPE, which are known to exhibit high TE in cell culture with low toxicity $(26,32)$.

Figure 5 (left) shows total gene knockdown data comparing the $K_{\mathrm{T}}$ of MVL5/DOPC-siRNA and DOTAP/DOPCsiRNA complexes at $\rho_{\text {chg }}=15$ as a function of $\Phi_{\mathrm{NL}}$ in the regime where the nonspecific knockdown shown in Figure 4 is relatively low (DOTAP/DOPE-siRNA data not shown because of large $K_{\mathrm{NS}}$ contribution). Complexes containing pentavalent MLV5 show a relatively high silencing efficiency over a broad range with $K_{\mathrm{T}} \approx 0.9$ for $0<$ $\Phi_{\mathrm{NL}}<0.5$. In contrast, $K_{\mathrm{T}}$ of DOTAP containing complexes remains relatively low and decreases from 0.6 to $\approx 0.5$ in the same range of $\Phi_{\mathrm{NL}}$.

To compare $\mathrm{CL}-$ siRNA behavior with prior observations for CL-DNA complexes, $K_{\mathrm{T}}$ for MVL5/DOPC-siRNA, DOTAP/DOPC-siRNA, and DOTAP/DOPE-siRNA complexes was examined at $\rho_{\text {chg }}=2.8$ (Figure 5, right), which corresponds to optimal transfection efficiency for DOTAP 

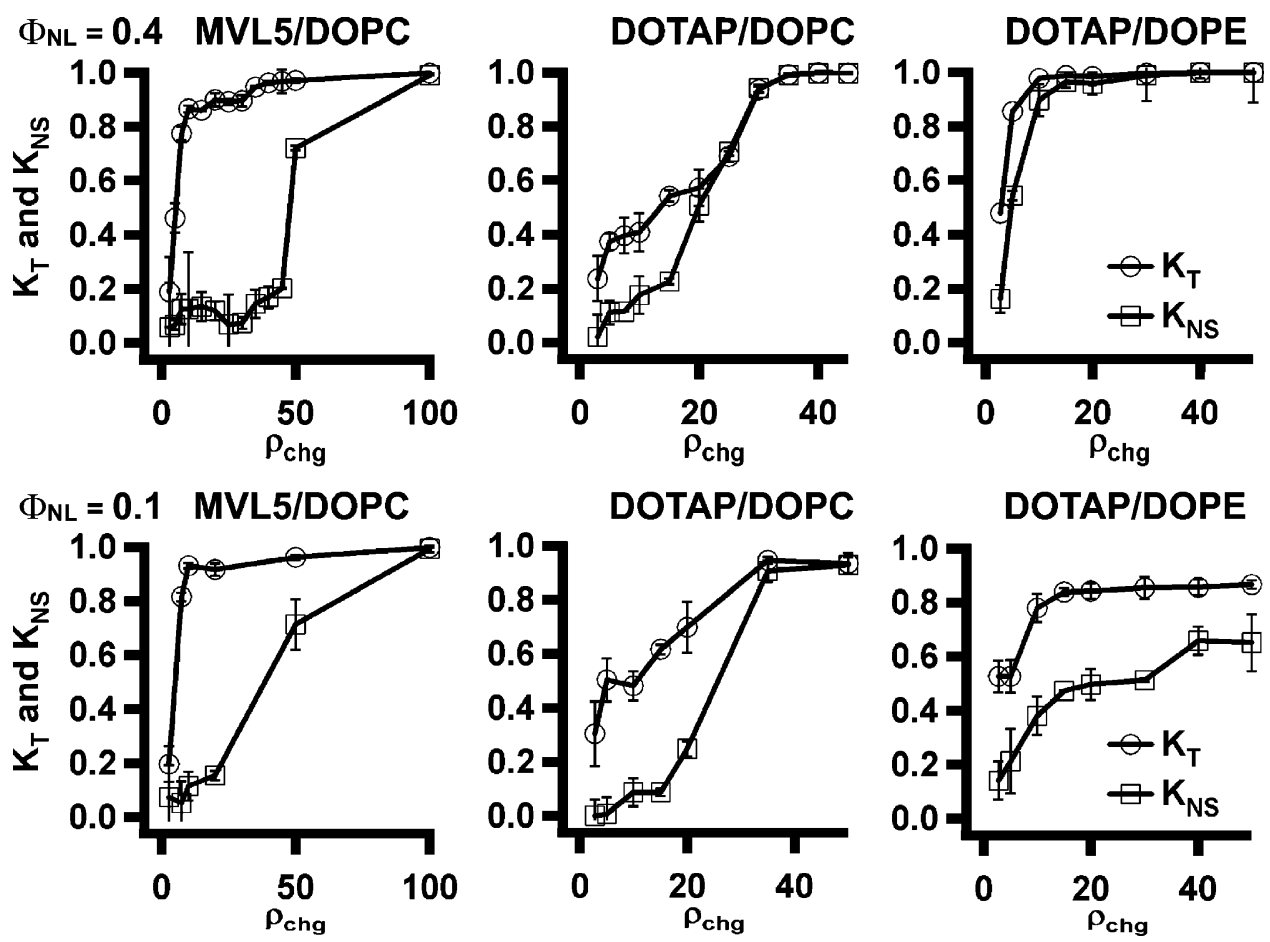

FIGURE 4: Total $\left(K_{\mathrm{T}}\right.$, open circles) and nonspecific ( $K_{\mathrm{NS}}$, open squares) gene knockdown versus $\rho_{\text {chg }}$ at $\Phi_{\mathrm{NL}}=0.4$ (top) and $\Phi_{\mathrm{NL}}=0.1$ (bottom) for MVL5/DOPC-siRNA (left), DOTAP/DOPC-siRNA (middle), and DOTAP/DOPE-siRNA (right) complexes targeting the luciferase mRNA in transfected L-cells.
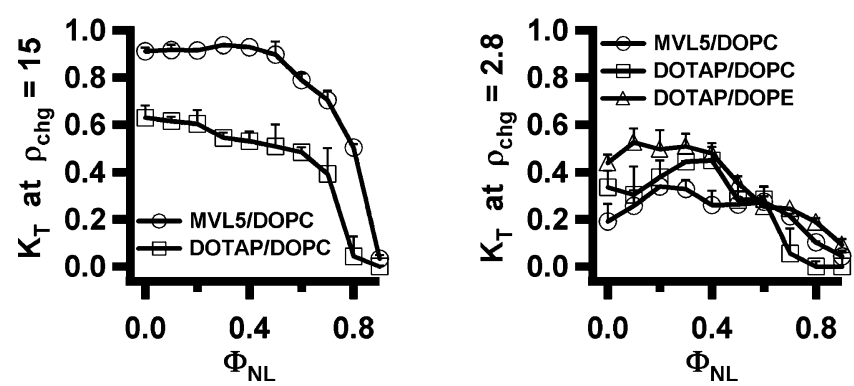

FIGURE 5: Total gene knockdown $\left(K_{\mathrm{T}}\right)$ with siRNA complexes targeting the luciferase mRNA in transfected mouse L-cells as a function of $\Phi_{\mathrm{NL}}$ at $\rho_{\mathrm{chg}}=15$ (left, MVL5/DOPC-siRNA, DOTAP/ DOPC-siRNA) and $\rho_{\text {chg }}=2.8$ (right, MVL5/DOPC-siRNA, DOTAP/DOPC-siRNA, and DOTAP/DOPE-siRNA).

containing CL-DNA complexes $(26,32)$ with very low cell toxicity (33). We observed that CL-siRNA complexes are generally inefficient at gene silencing at $\rho_{\text {chg }}=2.8$.

Cytotoxicity of Complexes Containing siRNA. Cytotoxicity of CL-siRNA complexes (MVL5/DOPC-siRNA, DOTAP/ DOPC-siRNA, DOTAP/DOPE-siRNA) as a function of $\Phi_{\mathrm{NL}}$ is shown in Figure 6 . The pink filled triangles $\left(\rho_{\mathrm{chg}}=\right.$ $10)$ and blue filled circles $\left(\rho_{\text {chg }}=50\right)$ represent toxicity data for cells incubated with complexes containing siRNA. The amount of siRNA in the complexes is constant for all data points. Also plotted are toxicity values measured when cells were incubated with corresponding amounts of cationic liposomes without siRNA (open maroon triangle and open green circle). Because the degree of cytotoxicity is comparable for cells incubated with either CL-siRNA complexes or cationic liposomes alone, its origin appears to be the lipid component of the complex.

At $\rho_{\text {chg }}=10$, only the DOTAP/DOPE - siRNA complexes and DOTAP/DOPE liposomes showed toxicity (Figure 6, right). For these systems, the toxicity exhibited a marked broad peak for $0.4<\Phi_{\mathrm{DOPE}}<0.8$. This is in contrast to the DOTAP/DOPC-siRNA (Figure 6, middle) and MVL5/ DOPC-siRNA (Figure 6, left) complexes and the corresponding liposomes, where toxicity was low for all $\Phi_{\text {DOPC }}$. The toxicity increase seen in the DOPE systems may result from an excessive fusion of DOPE containing complexes/ liposomes with the cell membrane, possibly creating pores and leading to membrane instability. At large $\Phi_{\mathrm{DOPE}}$, the toxicity decreased due to a reduced $\sigma_{\mathrm{M}}$, which decreases the interaction between the liposome/complex and the cell. In this context, it is important to note that no toxicity was observed upon incubating cells with only DOPC or DOPE liposomes (i.e., no cationic lipid) (data not shown), indicating that the cationic lipid was the enabling component for the toxicity trends in Figure 6.

For $\rho_{\text {chg }}=50$, we observed that siRNA complexes and cationic liposomes showed significant toxicity for all lipid combinations. Thus, the toxicity data correlated well with the measured nonspecific knockdown values $K_{\mathrm{NS}}$ (Figure 4) and supports the use of $K_{\mathrm{NS}}$ as an indicator for cell viability. The $20 \%$ increase in toxicity seen at $\rho_{\text {chg }}=50$ and $\Phi_{\mathrm{NL}}=$ 0 presumably must be due to the increased amount of CL. Also, at the fixed $\rho_{\text {chg }}=50$, it is likely that the toxicity of both the DOTAP/DOPE and DOTAP/DOPC samples is increasing with $\Phi_{\mathrm{NL}}$ as a result of increasing the total number of NLs and, thus, total lipid in the system. In the case of the MVL5/DOPC samples at $\rho_{\text {chg }}=50$, there was no increase in toxicity with $\Phi_{\text {DOPC }}$. MVL5 samples contain five times fewer NL at all points compared to the DOTAP samples, and it is reasonable to believe that this reduced NL content accounts for the lower toxicity. Furthermore, at $\rho_{\text {chg }}=50$, the toxicity for the DOTAP/DOPC and DOTAP/DOPE samples decreased at high $\Phi_{\mathrm{NL}}$, as explained above. However, the MVL5/DOPC samples did not show a decrease in toxicity at high $\Phi_{\mathrm{NL}}$, because MVL5 complexes and lipo- 

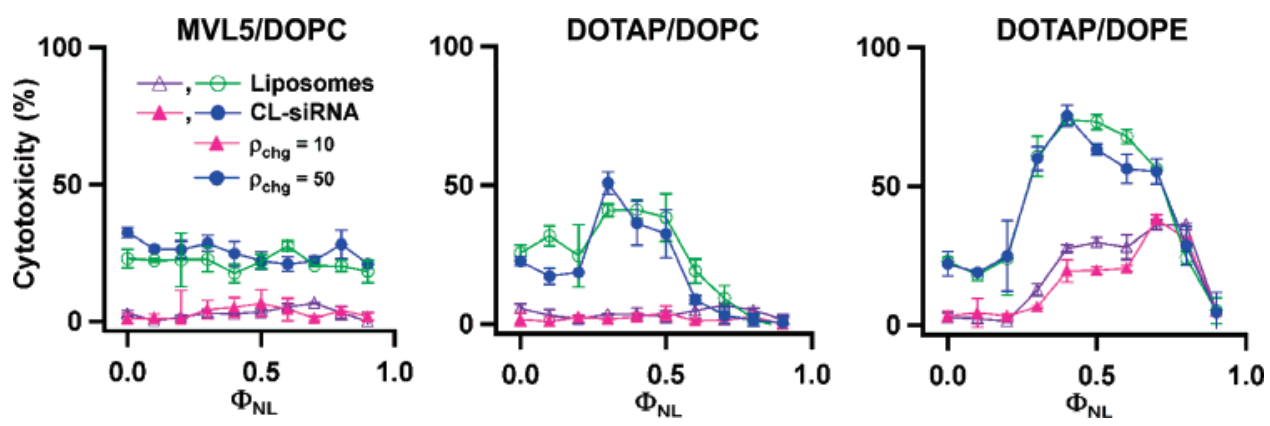

FIGURE 6: Cytotoxicity of CL-siRNA complexes (MVL5/DOPC-siRNA, DOTAP/DOPC-siRNA, DOTAP/DOPE-siRNA) targeting the luciferase mRNA in mouse L-cells and the corresponding cationic liposomes (without siRNA) as function of $\Phi_{\mathrm{NL}}$. For the complexes, the data are for $\rho_{\text {chg }}=10$ (filled triangle) and 50 (filled circle). The open maroon triangles and open green circles denote the cytotoxicity of cationic liposomes using the same amount of lipid as the complexes at $\rho_{\text {chg }}=10$ or 50 respectively. Cytotoxicity was measured by quantitating the amount of released lactate dehydrogenase from cells with damaged membranes.

somes at high $\Phi_{\text {DOPC }}$ still produced a significant amount of charge per area due to the multiple charges per head group. Therefore, a strong electrostatic interaction was maintained between MVL5 systems and the cells for all $\Phi_{\text {DOPC }}$.

The combined total knockdown $K_{\mathrm{T}}$, nonspecific knockdown $K_{\mathrm{NS}}$, and cytotoxicity data described here reveal that pentavalent MVL5/DOPC-siRNA complexes targeting the FF luciferase gene have a significantly higher silencing efficiency (with $K_{\mathrm{T}} \approx 0.9$ and $K_{\mathrm{NS}}<0.1$ ) and lower cell toxicity over a broader range of $\rho_{\text {chg }}$ and $\Phi_{\mathrm{NL}}$ when compared to the DOTAP/DOPC-siRNA complexes, with the latter not showing a regime with high $K_{\mathrm{T}}$ (approaching 1) and low $K_{\mathrm{NS}}$.

RNAi has revolutionized functional genomics and is currently being pursued as a therapeutic approach for a wide variety of diseases. Cationic lipids are widely used for the delivery of RNAi mediators such as short hairpin RNA expression plasmids and synthetically prepared siRNA duplexes. Herein, we provide new insights concerning the structural properties, delivery efficiency, and toxicity of CL vectors. We elucidated structural relationships between complexes of siRNA with DOTAP/DOPC, DOTAP/DOPE, and MVL5/DOPC and determined vector compositions that successfully deliver siRNA for RNAi by comparing the total knockdown $\left(K_{\mathrm{T}}\right)$, nonspecific knockdown $\left(K_{\mathrm{NS}}\right)$, and cytotoxicity of these delivery vectors.

While structural similarities were seen between CLsiRNA and CL-DNA complexes, differences did exist. siRNA complexes rapidly reached a structural equilibrium compared to DNA complexes and had a higher degree of local ordering while the siRNA layers existed in a liquidlike phase.

We found, for siRNA complexes, that a $\rho_{\text {chg }}$ larger than that used in DNA delivery was paramount in order to achieve efficient silencing with $K_{\mathrm{T}}$ approaching 1 and $K_{\mathrm{NS}}$ approaching zero. This $\rho_{\text {chg }}$ requirement resulted in considerably higher amounts of lipid in the complex, with subsequent biophysical and biological consequences. Factors contributing to the observed larger $\rho_{\text {chg }}$ include the following: First, the rotational and translational degrees of freedom associated with siRNA in the bulk is greater than that of long DNA, creating a larger barrier for siRNA complex formation (loss of entropy) as the siRNA shifts from the bulk state to the confined complexed state. Second, there is a difference in electrostatic adhesion energy per unit length between long DNA chains and short siRNA molecules adsorbed on the
CL membranes. The difference in adhesion energy arises because free DNA in solution contains bound counterions (34) which, upon adsorption to cationic membranes, are released back into solution. This contribution is about onehalf the adhesion energy, with the remaining one-half arising from an equal release of membrane bound counterions into solutions upon complexation (30). On the other hand, short siRNA is not expected to contain bound counterions (35) (but rather, counterions will be in its vicinity) decreasing the adhesion energy per unit length, compared to DNA, by about one-half.

Finally, differences in the electrostatic repulsion between DNA chains or siRNA molecules will further affect the packing efficiency in complexes. These free energy considerations are potentially creating siRNA-complex instability at low $\rho_{\text {chg. }}$. Increasing the chemical potential of the cationic lipids by increasing their concentration (larger $\rho_{\text {chg }}$ ) is thus needed to create stable, efficiently transfecting complexes.

We observed that the use of DOPE instead of DOPC as a neutral helper lipid did not improve silencing efficiency and, in fact, was toxic to cells when used in amounts required for efficient siRNA delivery. This result, again, is in striking contrast to prior findings obtained with optimized lipid vectors for DNA delivery, where the use of DOPE in place of DOPC can not only cause a structural phase change from $\mathrm{L}_{\alpha}$ to $\mathrm{H}_{\mathrm{II}}$ but also be beneficial to the transfection efficiency of DOTAP-based vectors in certain ranges of lipid composition. The structure of the complex may impact the delivery of siRNA; however, since the hexagonal phase is seen in CL-siRNA complexes with a high mole fraction DOPE, it is hard to distinguish whether observed transfection and toxicity effects are due to DOPE acting as a fusogenic helper lipid or due to the hexagonal complex structure DOPE induces.

Several key findings of our study emerged from the comparison of siRNA vectors containing multivalent $(5+)$ MVL5 versus monovalent DOTAP. MVL5-based vectors exhibited significantly improved $K_{\mathrm{T}}$ and a considerably lower $K_{\mathrm{NS}}$, and reduced toxicity when compared with DOTAPbased vectors. Our data also suggest that the $K_{\mathrm{NS}}$ and toxicity of CL delivery vectors depend on the amount of CL used. Of note, the increased valence of MVL5 allows for the formation of siRNA complexes with five times fewer lipid molecules than DOTAP and resulted in reduced toxicity.

In addition, we have seen that the toxicity of siRNA complexes to cells in culture is similar to that of correspond- 
ing liposomes and, therefore, is highly dependent on the type and amount of lipid used. These observations established the multivalent MVL5 as a superior transfection vector for siRNAs, and have implications for the optimal use of CL in therapeutic applications.

\section{ACKNOWLEDGMENT}

The X-ray diffraction work was carried out at the Stanford Synchrotron Radiation Laboratory (SSRL). The SSRL is supported by the Department of Energy.

\section{REFERENCES}

1. Fire, A., Xu, S. Q., Montgomery, M. K., Kostas, S. A., Driver, S. E., and Mello, C. C. (1998) Potent and specific genetic interference by double-stranded RNA in Caenorhabditis elegans, Nature 391, 806-811.

2. Napoli, C., Lemieux, C., and Jorgensen, R. (1990) Introduction of a Chimeric Chalcone Synthase Gene into Petunia Results in Reversible Co-Suppression of Homologous Genes in trans, Plant Cell 2, 279-289.

3. Jorgensen, R. A., Cluster, P. D., English, J., Que, Q. D., and Napoli, C. A. (1996) Chalcone synthase cosuppression phenotypes in petunia flowers: Comparison of sense vs antisense constructs and single-copy vs complex T-DNA sequences, Plant Mol. Biol. 31, 957-973.

4. McManus, M. T., and Sharp, P. A. (2002) Gene silencing in mammals by small interfering RNAs, Nat. Rev. Genet. 3, 73747.

5. Martinez, L. A., Naguibneva, I., Lehrmann, H., Vervisch, A., Tchenio, T., Lozano, G., and Harel-Bellan, A. (2002) Synthetic small inhibiting RNAs: efficient tools to inactivate oncogenic mutations and restore 53 pathways, Proc. Natl. Acad. Sci. U.S.A. 99, 14849-54.

6. Hammond, S. M., Caudy, A. A., and Hannon, G. J. (2001) Posttranscriptional gene silencing by double-stranded RNA, Nat. Rev. Genet. 2, 110-9.

7. Elbashir, S. M., Harborth, J., Lendeckel, W., Yalcin, A., Weber, K., and Tuschl, T. (2001) Duplexes of 21-nucleotide RNAs mediate RNA interference in cultured mammalian cells, Nature 411, 494-8.

8. Caplen, N. J., Parrish, S., Imani, F., Fire, A., and Morgan, R. A. (2001) Specific inhibition of gene expression by small doublestranded RNAs in invertebrate and vertebrate systems, Proc. Natl. Acad. Sci. U.S.A. 98, 9742-7.

9. Sioud, M. (2004) Therapeutic siRNAs, Trends Pharmacol. Sci. $25,22-8$.

10. Karagiannis, T. C., and El-Osta, A. (2005) RNA interference and potential therapeutic applications of short interfering RNAs, Cancer Gene Ther. 12, 787-95.

11. Hannon, G. J., and Rossi, J. J. (2004) Unlocking the potential of the human genome with RNA interference, Nature 431, 371-8.

12. Caplen, N. J. (2003) RNAi as a gene therapy approach, Expert Opin. Biol. Ther. 3, 575-86.

13. Paddison, P. J., Caudy, A. A., Bernstein, E., Hannon, G. J., and Conklin, D. S. (2002) Short hairpin RNAs (shRNAs) induce sequence-specific silencing in mammalian cells, Genes Dev. 16, 948-58.

14. Soutschek, J., Akinc, A., Bramlage, B., Charisse, K., Constien, R., Donoghue, M., Elbashir, S., Geick, A., Hadwiger, P., Harborth, J., John, M., Kesavan, V., Lavine, G., Pandey, R. K., Racie, T., Rajeev, K. G., Rohl, I., Toudjarska, I., Wang, G., Wuschko, S., Bumcrot, D., Koteliansky, V., Limmer, S., Manoharan, M., and Vornlocher, H.-P. (2004) Therapeutic silencing of an endogenous gene by systemic administration of modified siRNAs, Nature 432, $173-178$.

15. Lorenz, C., Hadwiger, P., John, M., Vornlocher, H.-P., and Unverzagt, C. (2004) Steroid and lipid conjugates of siRNAs to enhance cellular uptake and gene silencing in liver cells, Bioorg. Med. Chem. Lett. 14, 4975-4977.
16. Mahato, R. I., and Kim, S. W. (2002) Pharmaceutical Perspectives of Nucleic Acid-Based Therapeutics, Taylor and Francis, London and New York.

17. Huang, L., Hung, M.-c., and Wagner, E. (2005) Non-viral vectors for gene therapy, 2nd ed., Elsevier Academic Press, Amsterdam, Boston.

18. Ewert, K., Slack, N. L., Ahmad, A., Evans, H. M., Lin, A. J., Samuel, C. E., and Safinya, C. R. (2004) Cationic lipid-DNA complexes for gene therapy: Understanding the relationship between complex structure and gene delivery pathways at the molecular level, Curr. Med. Chem. 11, 133-149.

19. Ewert, K., Ahmad, A., Evans, H. M., and Safinya, C. R. (2005) Cationic lipid-DNA complexes for non-viral gene therapy: relating supramolecular structures to cellular pathways, Expert Opin. Biol. Ther. 5, 33-53.

20. Clark, P. R., and Hersh, E. M. (1999) Cationic lipid-mediated gene transfer: current concepts, Curr. Opin. Mol. Ther. 1, 158-76.

21. Chesnoy, S., and Huang, L. (2000) Structure and function of lipidDNA complexes for gene delivery, Annu. Rev. Biophys. Biomol. Struct. 29, 27-47.

22. Byk, G., Dubertret, C., Escriou, V., Frederic, M., Jaslin, G., Rangara, R., Pitard, B., Crouzet, J., Wils, P., Schwartz, B., and Scherman, D. (1998) Synthesis, activity, and structure-activity relationship studies of novel cationic lipids for DNA transfer, $J$. Med. Chem. 41, 229-35.

23. Boussif, O., Lezoualc'h, F., Zanta, M. A., Mergny, M. D., Scherman, D., Demeneix, B., and Behr, J. P. (1995) A versatile vector for gene and oligonucleotide transfer into cells in culture and in vivo: polyethylenimine, Proc. Natl. Acad. Sci. U.S.A. 92, 7297-301.

24. Thomas, C. E., Ehrhardt, A., and Kay, M. A. (2003) Progress and problems with the use of viral vectors for gene therapy, Nat. Rev. Genet. 4, 346-58.

25. Spagnou, S., Miller, A. D., and Keller, M. (2004) Lipidic carriers of siRNA: differences in the formulation, cellular uptake, and delivery with plasmid DNA, Biochemistry 43, 13348-56.

26. Ahmad, A., Evans, H. M., Ewert, K., George, C. X., Samuel, C. E., and Safinya, C. R. (2005) New multivalent cationic lipids reveal bell curve for transfection efficiency versus membrane charge density: lipid-DNA complexes for gene delivery, J. Gene Med. 7, 739-48.

27. Koltover, I., Salditt, T., Radler, J. O., and Safinya, C. R. (1998) An inverted hexagonal phase of cationic liposome-DNA complexes related to DNA release and delivery, Science 281, 78-81.

28. Lin, A. J., Slack, N. L., Ahmad, A., Koltover, I., George, C. X., Samuel, C. E., and Safinya, C. R. (2000) Structure and StructureFunction Studies of Lipid/Plamid DNA complexes, J. Drug Targeting 8, 13-27.

29. Ewert, K., Ahmad, A., Evans, H. M., Schmidt, H.-W., and Safinya, C. R. (2002) Efficient Synthesis and Cell-Transfection Properties of a New Multivalent Cationic Lipid for Nonviral Gene Delivery, J. Med. Chem. 45, 5023-5029.

30. Radler, J. O., Koltover, I., Salditt, T., and Safinya, C. R. (1997) Structure of DNA-cationic liposome complexes: DNA intercalation in multilamellar membranes in distinct interhelical packing regimes, Science 275, 810-814.

31. Subramanian, G., Hjelm, R. P., Deming, T. J., Smith, G. S., Li, Y., and Safinya, C. R. (2000) Structure of complexes of cationic lipids and poly(glutamic acid) polypeptides: A pinched lamellar phase, J. Am. Chem. Soc. 122, 26-34.

32. Lin, A. J., Slack, N. L., Ahmad, A., George, C. X., Samuel, C. E., and Safinya, C. R. (2003) Three-Dimensional Imaging of Lipid Gene-Carriers: Membrane Charge Density Controls Universal Transfection Behavior in Lamellar Cationic Liposome-DNA Complexes, Biophys. J. 84, 3307-3316.

33. Ewert, K. K., Evans, H. M., Bouxsein, N. F., and Safinya, C. R. (2006) Dendritic cationic lipids with highly charged headgroups for efficient gene delivery, Bioconjugate Chem. 17, 877-88.

34. Manning, G. S. (1969) Limiting Laws and Counterion Condensation in Polyelectrolyte Solutions. I. Colligative Properties, J. Chem. Phys. 51, 924.

35. Zimm, B. H., and Lebret, M. (1983) Counterion Condensation and System Dimensionality. J. Biomol. Struct. Dyn. 1, 461-471.

BI062138L 